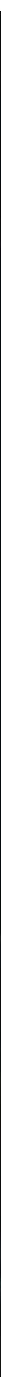

\title{
PROVING EINSTEIN RIGHT
}

\section{The committed team behind LIGO's detection of gravitational waves is contending with ongoing technical challenges of a mighty big laboratory.}

\section{BY KATHERINE BOURZAC}

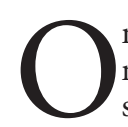
n 14 September 2015, David Shoemaker, a physicist at MIT, woke up to some incredible news. Detectors in the states of Washington and Louisiana had picked up ripples in space-time - gravitational waves - emanating from the collision of two black holes.

Shoemaker and about 1,000 of his colleagues at the Laser Interferometer Gravitational-Wave Observatory (LIGO) Scientific Collaboration checked and debated the data for months, publishing the results on 11 February 2016.
The LIGO detection was a physics landmark. It provided direct, observational evidence for a prediction made by Einstein more than 100 years ago in his theory of general relativity. It was also the first time astrophysicists witnessed black holes merging.

Since then, LIGO has announced several more gravity wave detections, also emanating from black-hole mergers, with their latest announcement of a fifth detection, this time of colliding neutron stars. And on 3 October 2017, three members of the LIGO team Barry Barish, Rainer Weiss and Kip Thorne - were awarded the Nobel Prize in Physics for their work in detecting the first waves.
The global fanfare surrounding these scientific breakthroughs has been the culmination of years of hard work. "The physics is really the easy part," says Shoemaker, now the spokesperson for the LIGO Scientific Collaboration. More complex was the delicate precision-engineering in designing, upgrading and now maintaining the facilities needed to detect black hole collisions more than a billion light years away. Add to that the more earthly challenges of getting a group of about 1,000 opinionated scientists in a dozen countries to work on a common project, interpret complex data, and agree on wording for a research paper. 
The next big task for project leaders is to figure out how to transition from a blue-sky experiment to an operational, data-producing astrophysical observatory. Engineers have begun the next round of upgrades, which brings new technical quandaries.

\section{CROSS-COUNTRY COLLABORATION}

Detecting gravitational waves from even the most catastrophic celestial events, such as black-hole or neutron-star collisions, requires exquisitely sensitive instruments.

In the 1960s, physicists, including MIT's Weiss, imagined using a laser interferometer to detect these wrinkles in space-time. A laser interferometer splits a beam of light and sends it down two perpendicular tubes. The light reflects off a mirror at the end of either tube, then recombines at a detector. When the lightwaves combine, they create an interference pattern, which adds up the peaks and troughs of the waves. Weiss reasoned that a passing gravitational wave would stretch and compress the space through which the beams of light travel, extending and shortening their path. This warping of space-time would appear as distortions in the interference pattern.

Groups at Caltech were also exploring the idea. In 1980, the National Science Foundation (NSF) funded prototype interferometers at Caltech and at MIT.

Nine years later, the two universities submitted a joint proposal for LIGO. In their design, interferometers with four-kilometre-long arms would be sited in two distant locations - one in Hanford, Washington, and the other in Livingston, Louisiana. The detectors were constructed at a great enough distance (roughly 3,000 kilometres) to eliminate any shared sources of distortion or 'noise' in the signal, such as seismic activity jostling the system.

The equipment and observatories would be operated by LIGO Laboratory, a collaboration between Caltech and MIT. These two institutions are among the top five US contributors to the authorship of physical sciences articles in the journals included in the index, collaborating on 209 papers in 2016. LIGO's activities and data analysis would be coordinated by the LIGO Scientific Collaboration, a much larger community of members from more than 100 institutions and 18 countries.

"IMAGINE WRITING A PAPER WITH 1,000 AUTHORS WHO ALL HAVE AN OPINION ABOUT WHAT SHOULD BE IN IT."
PHYSICAL STRENGTH

The majority of inter-state collaborations between Massachusetts and California are in the life sciences, but narrowing in on collaborative relationships between MIT and Caltech reveals a shift in the subject balance. The two institutions have co-authored the most papers in the physical sciences.

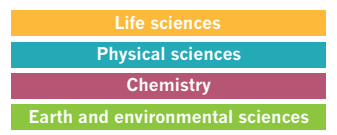

Massachusetts and California have established the most bilateral institutional partnerships in the life sciences in the Nature Index in 2016.

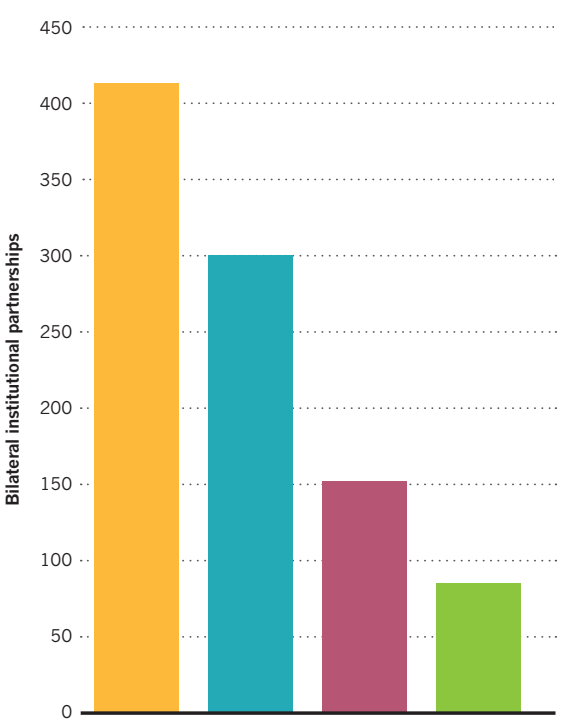

Collaborations between MIT on the east coast and Caltech on the west coast are dominated by the physical sciences. Collaboration score (CS) sums the fractional count of collaborative papers from the two partnering institutions.

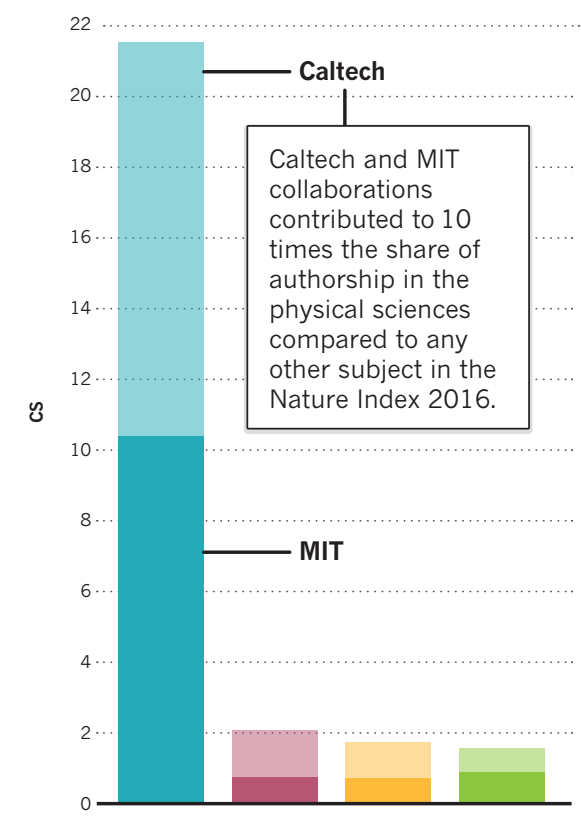

Building LIGO was unlike any other largescale physics undertaking, says Barish, a Caltech physicist who was the project's executive director from 1994 (when construction began) to 2005. Designers of telescopes and particle accelerators can draw on a legacy of experimental knowhow. Gravitational wave physicists were starting from scratch. "It was a huge extrapolation," says Barish. "Much of the technology had not been proven."

To detect gravitational waves, LIGO must measure minuscule changes in a light beam's distance travelled - far smaller than the diameter of an atom. This meant keeping the long steel tubes as empty of matter as possible to avoid wayward gas molecules from interfering with the light path. To ensure the cleanest signal possible, the team planned to create "the ultimate vacuum system," says Barish, in paired pipes, four kilometres long. Such a large vacuum had never been attempted. Engineers had to fit the tubes with doors to allow engineers to access the equipment inside.

The 340-millimetre-diameter mirrors at the ends of the tubes are "exquisite objects," says LIGO Lab chief engineer, Dennis Coyne, who is based at Caltech. To clean these mirrors, engineers have to climb into the vacuum chamber between runs, spread on a layer of polymer, and peel it off, bringing any dust with it.

Success was hard earned. The team ran the first phase of observations through 2010 - and saw no astronomical signals. No characteristic gravitational wave squiggles from colliding black holes or neutron stars, no hum from the Big Bang, just noise. LIGO picked up cars driving by, engineers' footsteps, small earthquakes, and even the vibrations of the guitar-string-like suspension wires holding up the mirrors.

The team upgraded the detectors over five years, replacing the vibrating steel wires with quieter silica fibres, as well as other adjustments to compensate for systematic errors. LIGO detected gravitational waves in September 2015 thanks to what Barish calls "the world's best shock absorbers". The team suspended the mirrors below a series of three weights that would soak up vibrations, like shock absorbers. Another detection was made in December 2015, and again in January and August 2017.

Getting over all these hurdles has brought the LIGO engineers and scientists together. "We're very close-knit," says Shoemaker. The group makes decisions as a team - in some cases, a very large team. "Imagine writing a paper with 1,000 authors who all have an opinion about what should be in it," says executive director of LIGO Lab, David Reitze. Conference calls routinely happen at 7 am California time to include the European groups; dedicated members in Australia must dial in at midnight.

\section{NEWFRONTIERS}

Now that LIGO has shown it is possible to detect gravitational waves, the team hopes to do it much more routinely - to become a 
MIT's David Shoemaker says the physics was

4. the easy part of getting LIGO up and running. The engineering and collaborativelogistics were much harder to overcome.

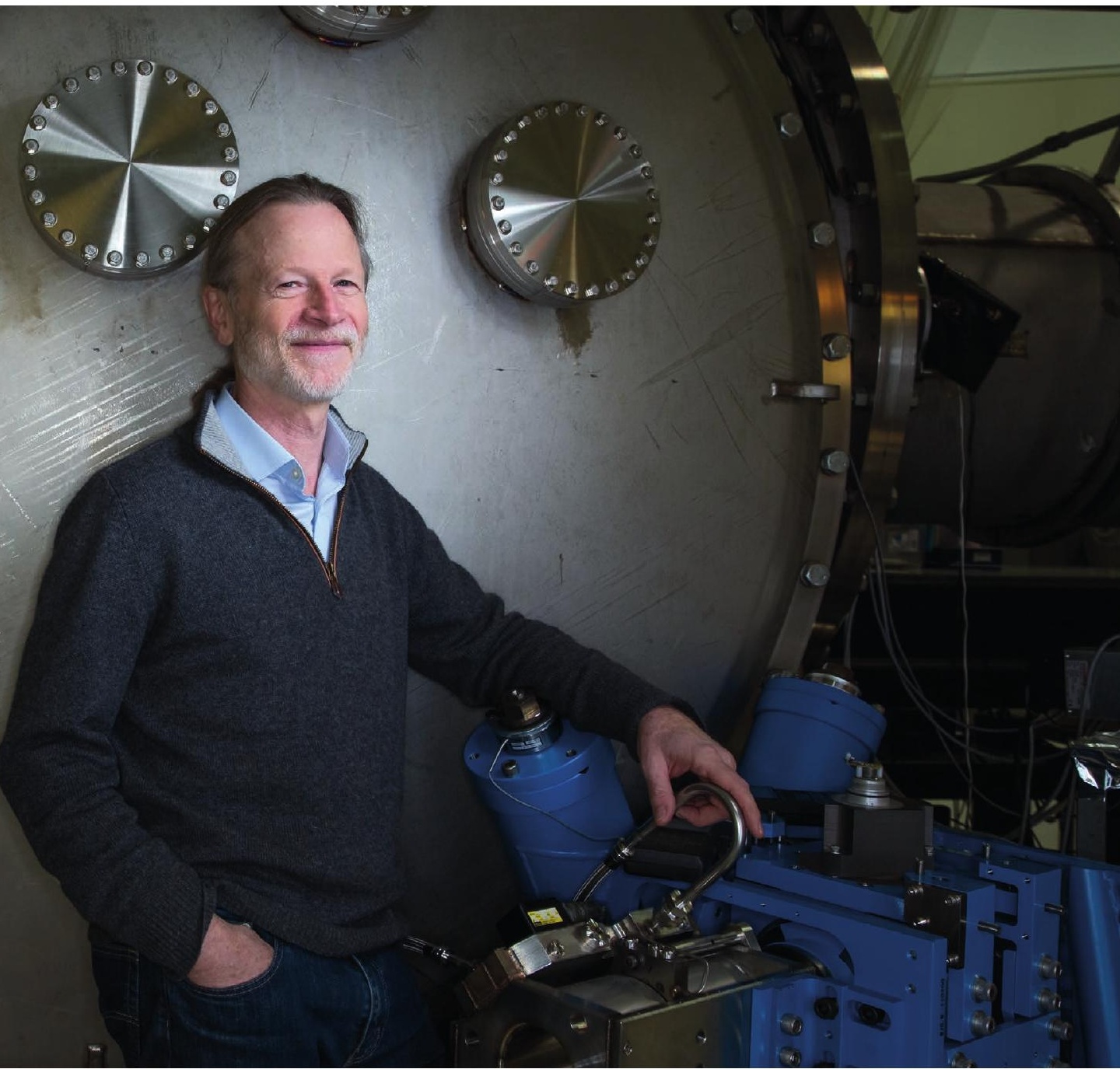

gravitational wave observatory, akin to a large telescope. This transition from ambitious physics experiment to everyday observatory comes with its own problems, says Shoemaker.

LIGO was designed with upgrades planned, and engineers want to keep making them. The payoff for physicists will be con-siderable. The deeper into the Universe they can see, the greater the likelihood of observing astronomical phenomena such as a spiralling pair of neutron stars. The detectors will soon go offline again until autumn 2018 for more upgrades.

LIGO's reach is currently limited by the power of the laser and the quality of the vacuum. So far, the lasers have not been turned to their full power, in part due to problems with scattered light reflecting off the tube and re-entering the beam. Engineers plan to add a protective layer to the tube walls that will absorb scattered light. They are also working on long-term strategies to improve sensitivity even further, including adopting a cutting-edge quantum optics technique called light squeezing that could make the signal clearer. So far this work is in theoretical stages, but the group

\section{"I AM EXCITED TO BE WOKEN IN THE MIDDLE OF THE NIGHT TO SEE WIGGLES THAT DON'T LOOK LIKE MODELS OF ANYTHING."}

is working on how it could be implemented at the LIGO observatories.

Some stakeholders object to the breaks. Funders want the observatory they paid for running, and so do astrophysicists who want to keep searching for signals. Black holes are considered to be the best laboratory for studying general relativity, and physicists have only just gained access to them through LIGO.
Despite these objections, some upgrades can't be delayed. The facilities need new roofs. The infrastructure, including the steel pipes housing the laser beams, is 20 years old, and the quality of the vacuum is degrading.

The Louisiana site has problems. Tiny holes are appearing along the tubes. "It's a 10,000 cubic-metre vacuum system, and finding and repairing these leaks is a challenge," says Reitze. Their hypothesis is that the steel might be corroding due to contact with mouse urine and with the chlorine from black widow spider poison. Wasps build their nests on the tubes, and put the spiders inside to feed their young. In probing the secrets of the Universe, says Reitze, "we're up against nature."

LIGO participants dream of less mundane concerns: what will they do when they detect a signal that they know is a gravitational wave, but that doesn't look like anything that theorists have predicted would emanate from a known astronomical phenomenon? "I am excited to be woken up in the middle of the night to see wiggles that don't look like our models of anything," says Shoemaker. 